\title{
Phytochemistry, Toxicology, and Pharmacological Properties of Origanum elongatum
}

\author{
Balahbib Abdelaali $\left(\mathbb{D},{ }^{1}\right.$ Naoual El Menyiy $\mathbb{D}^{2},{ }^{2}$ Nasreddine El Omari $\left(\mathbb{D},{ }^{3}\right.$ Taoufiq Benali, ${ }^{4}$ \\ Fatima-Ezzahrae Guaouguaou, ${ }^{5}$ Najoua Salhi, ${ }^{6}$ Hanae Naceiri Mrabti $\mathbb{D}^{6}{ }^{6}$ \\ and Abdelhakim Bouyahya $\mathbb{D D}^{7}$ \\ ${ }^{1}$ Laboratory of Biodiversity, Ecology and Genome, Mohammed V University in Rabat, Rabat, Morocco \\ ${ }^{2}$ Laboratory of Physiology, Pharmacology and Environmental Health, University Sidi Mohamed Ben Abdellah, Fez, Morocco \\ ${ }^{3}$ Laboratory of Histology, Embryology, and Cytogenetic, Mohammed V University in Rabat, Rabat, Morocco \\ ${ }^{4}$ Environment and Health Team, Polydisciplinary Faculty of Safi, Cadi Ayyad University, Marrakech, Morocco \\ ${ }^{5}$ Mohammed V University in Rabat, LPCMIO, Materials Science Center (MSC), Ecole Normale Supérieure, Rabat, Morocco \\ ${ }^{6}$ Laboratory of Pharmacology and Toxicology, Faculty of Medicine and Pharmacy, Mohammed V University in Rabat, BP 6203, \\ Rabat, Morocco \\ ${ }^{7}$ Laboratory of Human Pathologies Biology, Department of Biology, Mohammed V University in Rabat, Rabat, Morocco
}

Correspondence should be addressed to Abdelhakim Bouyahya; boyahyaa-90@hotmail.fr

Received 11 October 2020; Revised 20 April 2021; Accepted 7 June 2021; Published 17 June 2021

Academic Editor: Roberto K. N. Cuman

Copyright (c) 2021 Balahbib Abdelaali et al. This is an open access article distributed under the Creative Commons Attribution License, which permits unrestricted use, distribution, and reproduction in any medium, provided the original work is properly cited.

\begin{abstract}
Origanum elongatum L. is an endemic aromatic and medicinal plant. This work reports previous studies on O. elongatum concerning its taxonomy, botanical description, geographical distribution, bioactive compounds, toxicology, and biological effects. Chemical analyses showed that $O$. elongatum contains different chemical compounds, in particular volatile compounds. Pharmacological investigations showed that volatile compounds and extracts from $O$. elongatum exhibit different pharmacological properties, such as antibacterial, antifungal, antiviral, antioxidant, vasodilator, corrosion inhibitor, and hepatoprotective effects. Moreover, toxicological reports revealed the safety of this species. The pharmacological effects of O. elongatum could be correlated with the main compounds, which exhibit different pharmacological properties with numerous mechanism insights.
\end{abstract}

\section{Introduction}

Origanum elongatum (Bonnet) Emberger et Maire is an endemic aromatic and medicinal species of Morocco. It is a medicinal plant of the Lamiaceae family, a perennial herb of the Origanum genus. It is distributed in the wild species and is limited to the northeast (NE) of Morocco and extends from the Middle Atlas to the Rif Mountains ranges, mainly at high altitude on the mountains (the mountain of Tazekka and the mountain of Bouyablane).

Phytochemical investigations showed that O. elongatum contains several classes of bioactive compounds, including terpenoids, flavonoids, oxygenated compounds, hydrocarbon compounds, and phenolic compounds [1-8]. The main volatile compounds of this species are carvacrol, thymol, linalool, and limonene. Chemical analysis showed that the chemical composition is different between several published studies depending on the plant part and the collection site.

Pharmacological reports showed that extracts and essential oils (EOs) of O. elongatum exhibited various biological activities such as antibacterial [3, 4, 6, 9-11], antifungal [3, 12], antiparasitic [5, 13-16], antiviral [6], antioxidant [1], vasodilator [10,17], corrosion inhibitor [18], and hepatoprotective effects [19]. These biological effects are certainly due to the chemical composition of O. elongatum, in particular the main compounds such as carvacrol, linalool, and thymol. Indeed, literature reports revealed that these compounds possess several pharmacological effects. 
Moreover, the toxicological investigations showed that O. elongatum caused a slight change in behavior with loss of appetite and temporary sedation without any change in pathophysiological and neurological activity and $\mathrm{LD}_{50}$ was greater than $3000 \mathrm{mg} / \mathrm{kg}$ [19].

This review is designed to explore all previous studies on O. elongatum L. in terms of taxonomy, botanical description, geographic distribution, ethnobotanical prospecting, and toxicology and all the investigations on the biological activities of the different parts of this plant, and we will summarize the list of all phytochemicals isolated and identified from the extracts or EOs of this plant. This work aims to provide a scientific basis for further studies and the development of medicinal agents from O. elongatum.

\section{Research Methodology}

The collection of data about Origanum elongatum concerned its botanical description, taxonomy, destruction, phytochemistry, and biological properties. Numerous databases such as Google Scholar, Web of Science, Scopus, ScienceDirect, SpringerLink, Wiley Online, SciFinder, and PubMed were consulted to collect publications about O. elongatum. The collected data have covered all years. The collected articles were organized in tables, analyzed, and highlighted in this review according to each field. The chemical compounds of $\mathrm{O}$. elongatum were PubChem database and their chemical structures were drawn using ChemDraw Pro 8.0 software.

\section{Results and Discussion}

3.1. Botanical Description. Origanum elongatum (Bonnet) Emberger et Maire is an endemic species of Morocco. It is a woody perennial, which stems up to $90 \mathrm{~cm}$. Its stems are erect, light or dark brown (purplish) and at the bases hirsute (hairs c. $1.5 \mathrm{~mm}$ long), otherwise, glabrous, and often glaucous leaves. Branches of the first order are present, in the upper $1 / 3-1 / 2$ of the stems, up to 15 pairs per stem, $4 \mathrm{~cm}$ long; branches of the second order sometimes present; those of the third order seldom so. All branches entirely or for the greater part consist of spikes. Leaves up to 30 pairs per stem are shortly petiolate in the lower part to subsessile in the upper part (petioles up to $5 \mathrm{~mm}$ long), ovate or oval, margins entire, tops obtuse, $10 \mathrm{~mm}$ long, $8 \mathrm{~mm}$ wide, somewhat leathery, light green or purplish, often glaucous, glabrescent (pilose to glabrous; hairs c. $1.2 \mathrm{~mm}$ long), sessile glands up to 1600 per $\mathrm{cm}^{2}$. Spikes are very loose and tenuous, $40 \mathrm{~mm}$ long, c. $3 \mathrm{~mm}$ wide. Bracts are 10 pairs per spike, \pm lanceolate, tops acute, $3 \mathrm{~mm}$ long, $1 \mathrm{~mm}$ wide, glabrous or pilosellous, green, often glaucous. Flowers are subsessile. Calyces are $3.5 \mathrm{~mm}$ long, outside glabrous or pilosellous; teeth $1 \mathrm{~mm}$ long. Corollas are $6 \mathrm{~mm}$ long, pink, outside pilosellous; upper lips divided, for $c .1 / 5$, into $2, c .0 .3 \mathrm{~mm}$ long lobes; lower lips divided, for c. 3/5, into 3, somewhat unequal, $1 \mathrm{~mm}$ long lobes. Staminal filaments are up to 2 and $3.5 \mathrm{~mm}$ long. Styles are up to $8 \mathrm{~mm}$ long [20].
3.2. Taxonomy and Geographic Distribution. Origanum is derived from two Greek words, "oros" which means mountain and "ganos" which means shine; this word would mean "ornament of the mountains" [21]. The appearance of the French term was in the 13th century, European (Origanum sp.) and Mexican (Lippia sp.) "oregano." The name "Oregano" is commonly used around the world to define a spicy aroma and flavor [22].

The genus Origanum belongs to the Lamiaceae family and the Nepetoideae subfamily. The taxonomic point of view was completely revised by Dr. JH Ietswaart in 1980 [20]. In this work, Ietswaart divided the genus into 3 groups, 10 sections, grouping in total 38 species (one with 6 subspecies and another with 3 varieties), and 17 hybrids. This classification was based on the morphological characters of the plant (length of the stem, number of branches, and shape of the leaves) [20].

The flora of Morocco includes five taxa of Origanum, of which two (O. compactum and O. vulgare subsp. virens) are considered to be Ibero-Moroccan taxa and the other three taxa (O. elongatum, O. grosii, and O. fontqueri) are endemic in Morocco [2]. Due to their very similar morphologies, these three Moroccan steno-endemics are subject to taxonomic confusion. According to some databases [23], O. grosii and O. $\times$ fontqueri are considered to be synonyms of O. elongatum. However, Ietswaart [20] described O. grosii and $O$. elongatum as two different Origanum species according to their morphological characters, the length of the stems, ears, and leaves of O. Elongatum being larger than those of O. Grosii, but with O. grosii having a longer and wider bract than $O$. elongatum, while the hybrid, $O . \times$ fontqueri, is not described in the Ietswaart classification [20]. On the other hand, O. elongatum and O. grosii show obvious morphological differences from O. compactum (section Prolaticorolla Ietsw.) and from O. vulgare subsp. virens (section Origanum L.).

Origanum elongatum (Bonnet) Emb. \& Maire belongs to the Elongatispica section (Section 7) of group C [20]. It is recognized in Morocco by its common Arabic name "Zaatar." The geographical distribution of the wild species is limited to the NE of Morocco and extends from the Middle Atlas to the Rif Mountains ranges, mainly at high altitude on the mountains (the mountain of Tazekka and the mountain of Bouyablane) [20].

3.3. Ecological Factors. The wild species of O. elongatum grows at altitudes between 400 and $1500 \mathrm{~m}$ [24]; it abounds in open forests, rockeries, and mountain matorrals, on siliceous substrates and deep and well-drained soils. It is characterized by a fairly significant bioclimatic plasticity ranging from semiarid to per humid. The most favorable vegetation stages for this species are the thermo-Mediterranean and the meso-Mediterranean [25]. These oregano flowers from June to October [20] are known for their white inflorescence attached to vertical stems [24]. The abundance of their inflorescences, their lightness, and the sequence of flowering provide an ornamental interest to this species [20]. O. elongatum grows readily in temperate continental 
climates and grows rapidly, but with limited development in size. However, harvesting is possible in the first year but with a low yield of dry matter [7]. The germination of O. elongatum seeds is extremely affected by abiotic factors, such as temperature, salinity stress, and $\mathrm{pH}$. Thus, a temperature of $20^{\circ} \mathrm{C}$, a pH of 6 , and a salinity of $1 \mathrm{~g} / \mathrm{L}$ constitute the optimal conditions for germination of this species [26].

3.4. Phytochemistry. The phytochemical analysis of O. elongatum extracts and EOs revealed the presence of a set of compounds, which are summarized in Table 1. Terpenoids were among the chemical classes dominating in O. elongatum EOs (Figure 1).

The methanol extract and the ethyl acetate extract from the leaves of $O$. elongatum are rich in phenolic compounds. The total phenol content of these extracts is $153.22 \pm 2.67 \mathrm{mg}$ GAE/g of methanol extract and $130 \pm 3.0567 \mathrm{mg}$ GAE/g of ethyl acetate extract. However, the flavonoid content is $4.83 \pm 0.72 \mathrm{mgEQ} / \mathrm{g}$ of ethyl acetate extract and $5.02 \pm 0.26$ $\mathrm{mgEQ} / \mathrm{g}$ of methanol extract [17].

The analysis by gas chromatography of O. elongatum EO from Morocco shows that it consists of 11 compounds, of which 3 are in the majority; carvacrol (60.42\%), p-cymene (13.9\%), and $\gamma$-terpinene (9.4\%) [1].

In the same country (Morocco), 28 compounds were identified in O. elongatum EO, including carvacrol, thymol, and $p$-cymene, constituting the majority compounds. Additionally, limonene, thymoquinone, and thymohydroquinone have been reported in some EOs [2].

The main compounds identified in O. elongatum EO are carvacrol (63.06\%), $\gamma$-terpinene (15.99\%), p-cymene $(9.51 \%)$, and other compounds, with appreciable percentages such as $\alpha$-phellandrene, caryophyllene, and $\alpha$-pinene [3].

On the other hand, a previous study [4] showed that the chemical profile of $O$. elongatum EO shows the predominance of oxygenated compounds (65.14\%), followed by hydrocarbon compounds $(28.02 \%)$, knowing that thymol is the major compound with $63.44 \%$. These results confirm the findings of [5], which showed that the main constituents found in the aerial parts of O. elongatum EO are carvacrol (67.34\%), $\gamma$-terpinene (3.29\%), p-cymene (3.62\%), and thymol (1.79\%). However, in 2013, Moussaoui et al. [6] showed that the main constituents identified in the O. elongatum EO are carvacrol (40.12\%), thymol (14.24\%), p-cymene (16.19\%), and $\gamma$-terpinene (13.48\%).

Chromatographic analysis of the seeds of O. elongatum EO revealed the richness of the chemical composition predominated by carvacrol with a percentage of $79.2 \%$, followed by $\gamma$-terpinene (3.7\%), p-cymene (5.2\%), and linalool $(2.4 \%)[7,8]$.

3.5. Pharmacological Studies. EOs and extracts from O. elongatum showed different pharmacological properties such as antibacterial, antiparasitic, anticancer, and antioxidant effects (Figure 2). In the following part, all of these biological activities will be discussed.

3.6. Antibacterial Activity. Several studies showed the antibacterial efficacy of different EOs or extracts from different
O. elongatum parts [3, 4, 6, 9-11]. Table 2 summarizes all the studies evaluating the antimicrobial activity of $O$. elongatum extracts and EOs.

Bouharb et al. [9] evaluated in vitro the antibacterial activity of two extracts (aqueous and ethanolic) of $O$. elongatum leaves, from the Zerhoun region (central Morocco), on the growth of six strains of Pseudomonas aeruginosa (P, P3, P65, P381, P2, and P5), using the agar diffusion method and the broth macrodilution method of the active extract. The screening test revealed that $O$ elongatum aqueous extract was more active than the ethanolic extract, with zones of inhibition ranging from 9.33 to 11.83 and 8.33 to $11 \mathrm{~mm}$, respectively. Douhri et al. [19] also studied the antibacterial activity in vitro of ethanolic extracts of O. elongatum leaves. The results showed a very important antimicrobial effect against Escherichia coli $(30.33 \pm 2.51 \mathrm{~mm})$. Moreover, El Harsal and colleagues evaluated the antimicrobial activity of the volatile fractions extracted from the hydrosol (DVF) and of EOs from $O$. elongatum aerial parts growing in northern Morocco against four bacterial strains; E. coli ATCC 25922, E. coli K12, S. aureus ATCC 25923, and B. subtilis DCM 6633. They found that the antibacterial effect of DVF was significantly higher than that of total EO. The DVF was active against all the studied bacteria; the strongest effect was observed on B. subtilis DCM 6633 with a large inhibition zone $(41.0 \pm 2.6 \mathrm{~mm})$. Also, the total EO of O. elongatum was highly active against $S$. aureus ATCC 25923, B. subtilis DCM 6633, and E. coli ATCC 25922, with inhibition zones ranging from 21.3 to $24.6 \mathrm{~mm}$, while a moderate effect was observed against E. coli $\mathrm{K} 12$ [4].

In another study, the O. elongatum EO extracted from flowering tops and that extracted from leaves were tested against five microorganisms (Salmonella S64, Salmonella CECT 915, Listeria monocytogenes CECT4031, L. monocytogenes L23, and E. coli O157: H7 CECT4267), using the disk-diffusion assay and the microtitration assays. Additionally, the EOs showed the highest activities against the microorganisms tested, in particular against Salmonella and L. monocytogenes with zones of inhibition varying between $21.67 \pm 0.58 \mathrm{~mm}$ and $34.33 \pm 4.04 \mathrm{~mm}$. The moderate activity was recorded against $E$. coli, with zones of inhibition from $14.33 \pm 2.52$ to $19.67 \pm 1.15 \mathrm{~mm}$ [6]. Furthermore, a concentration of $0.06 \%$ of $O$. elongatum EO, extracted from flowering tops, showed a significant increase in the growth of total mesophilic aerobic flora (FMAT) [10]. In addition, the antibacterial activity of the $O$. elongatum EO (aerial part flowering) was tested against Staphylococcus aureus, $P$. aeruginosa, and $E$. coli. Therefore, an important inhibitory activity against all the strains tested was observed, with an inhibition diameter between 9.33 and $35.67 \mathrm{~mm}$ and high efficacy against E. coli and S. aureus [3].

3.7. Antifungal Activity. O. elongatum is one of the plants with antifungal properties [3, 12]. Indeed, several studies have evaluated these properties in O. elongatum EOs (Table 3). The antifungal activity of the essential oil of 
TABLE 1: Chemical composition of extracts and essential oils of O. elongatum.

\begin{tabular}{|c|c|c|c|c|c|}
\hline Country & Part & $\begin{array}{l}\text { Extracts/essential } \\
\text { oils }\end{array}$ & $\begin{array}{l}\text { Compounds } \\
\text { groups }\end{array}$ & Compounds & References \\
\hline Morocco & Leaves & $\begin{array}{l}\text { Ethyl acetate and } \\
\text { methanol extracts }\end{array}$ & $\begin{array}{l}\text { Total } \\
\text { polyphenols } \\
\text { Flavonoids }\end{array}$ & - & [17] \\
\hline Morocco & $\begin{array}{l}\text { Leaves and } \\
\text { flowering tops }\end{array}$ & Essential oil & Terpenoids & $\begin{array}{c}\alpha \text {-Thujene, } \beta \text {-myrcene, } p \text {-cymene, } \gamma \text {-terpinene, linalool, } \\
\text { terpinene- } 4 \text {-ol, thymol, carvacrol, } \beta \text {-caryophyllene, } \\
\beta \text {-bisabolene, and caryophyllene oxide }\end{array}$ & {$[1]$} \\
\hline Morocco & Aerial parts & Essential oil & Terpenoids & $\begin{array}{l}\text { Carvacrol, thymol, } p \text {-cymene, } \alpha \text {-terpinene, limonene, } \\
\text { thymoquinone, and thymohydroquinone }\end{array}$ & {$[2]$} \\
\hline Morocco & Aerial parts & Essential oil & Terpenoids & $\begin{array}{l}\text { Carvacrol, } \gamma \text {-terpinene, } p \text {-cymene, } \alpha \text {-phellandrene, } \\
\text { caryophyllene, } 3 \text {-carene, and } \alpha \text {-pinene }\end{array}$ & {$[3]$} \\
\hline Morocco & Aerial parts & Essential oil & Terpenoids & Thymol, $\gamma$-terpinene, and $p$-cymene & {$[4]$} \\
\hline Morocco & Aerial parts & Essential oil & Terpenoids & Carvacrol, thymol, $\gamma$-terpinene, and $p$-cymene & {$[5]$} \\
\hline Morocco & $\begin{array}{l}\text { Leaves and } \\
\text { flowering tops }\end{array}$ & Essential oil & Terpenoids & Carvacrol, thymol, $\gamma$-terpinene, and $p$-cymene & {$[6]$} \\
\hline Morocco & Seeds & Essential oil & Terpenoids & Carvacrol, thymol, $\gamma$-terpinene, $p$-cymene, and linalool & {$[8]$} \\
\hline Morocco & Seeds & Essential oil & Terpenoids & Carvacrol, $p$-cymene, $\gamma$-terpinene, and linalool & {$[7]$} \\
\hline
\end{tabular}

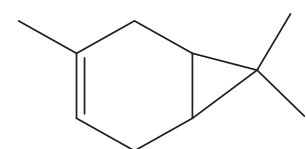

1: 3-carene

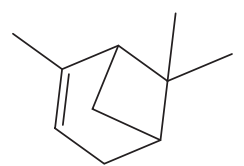

5: alpha-pinene<smiles>CC(C)=CCC=C(C)C1CC=CCC1</smiles>

9: beta-Bisabolene

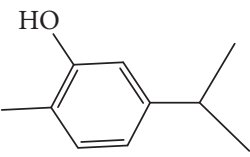

13: carvacrol

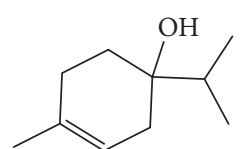

2: terpinen-4-ol

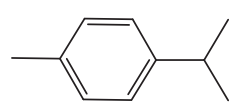

6: $p$-cymene

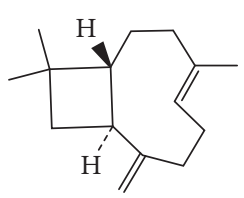

10: beta-caryophyllene

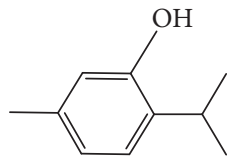

14: thymol

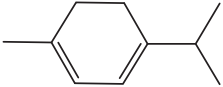

3: alpha-terpinene

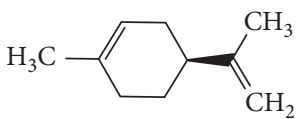

7: limonene

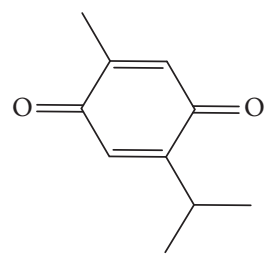

11: thymoquinone

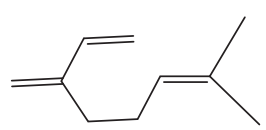

15: beta-Myrcene

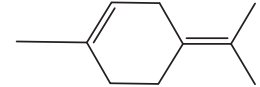

4: gamma-terpinene

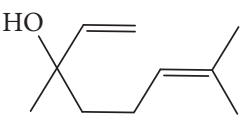

8: linalool

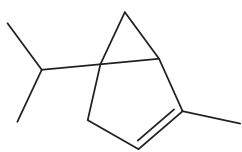

12: alpha-thujene

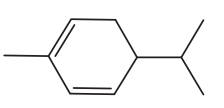

16: alpha-phellandrene

FIgURE 1: Chemical structures of terpenoids identified in O. elongatum EOs.

O. elongatum aerial parts was tested and evaluated by the microdilution method against three strains of fungi: Candida, Aspergillus, and Rhizopus [12]. Therefore, all Candida strains showed marked sensitivity to the essential oil. The Rhizopus strain was less sensitive, whereas for Aspergillus, this oil showed an effect only on tree strains. In another study, the antifungal activity of $O$. elongatum EO was evaluated by the agar plug diffusion method, which consequently showed promising results against Aspergillus brasiliensis (no measurable zone of inhibition) and Candida albicans $(33.67 \pm 0.33 \mathrm{~mm})$ [3].

3.8. Antiparasitic Activity. Many species of the genus Origanum have shown antiparasitic activities [13-15]. Moreover, the antiparasitic effect of $O$. elongatum was 


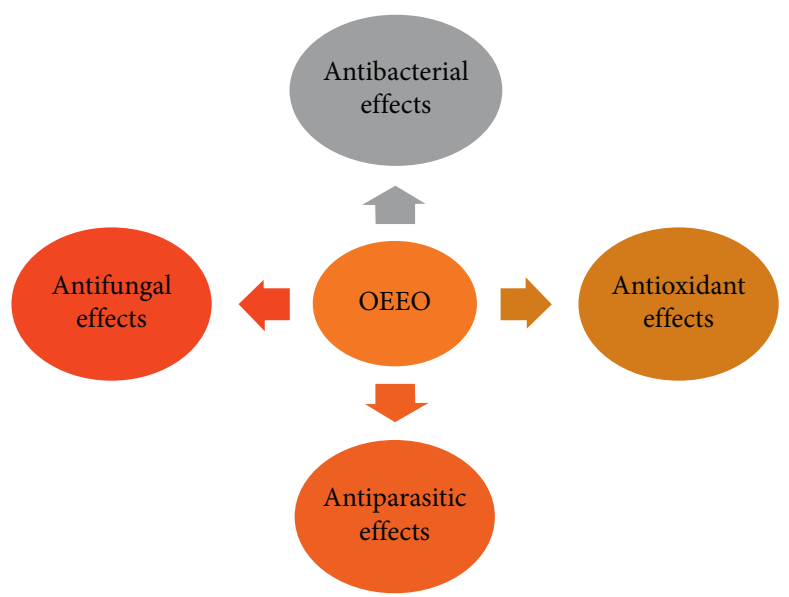

Figure 2: Pharmacological properties of O. elongatum.

TABle 2: Antibacterial effects of O. elongatum.

\begin{tabular}{|c|c|c|c|c|c|}
\hline Use part & Extracts & Used method & Tested strains & Key results & References \\
\hline Leaves & Aqueous extract & $\begin{array}{l}\text { Method of diffusion in solid } \\
\text { medium } \\
\text { Macromethod of dilution in } \\
\text { liquid medium }\end{array}$ & $\begin{array}{l}\text { P } \\
\text { P3 } \\
\text { P65 } \\
\text { P381 } \\
\text { P2 } \\
\text { P5 } \\
\text { P } \\
\text { P3 } \\
\text { P65 } \\
\text { P381 } \\
\text { P2 } \\
\text { P5 }\end{array}$ & $\begin{array}{c}- \\
\Phi=10 \pm 0.8 \mathrm{~mm} \\
\Phi=11 \pm 0.8 \mathrm{~mm} \\
\Phi=8.5 \pm 0.4 \mathrm{~mm} \\
\Phi=9 \pm 0.8 \mathrm{~mm} \\
\Phi=8.33 \pm 0.8 \mathrm{~mm} \\
\Phi=11.66 \pm 0.47 \mathrm{~mm} \\
\Phi=11 \pm 0.95 \mathrm{~mm} \\
\Phi=11.83 \pm 0.23 \mathrm{~mm} \\
\Phi=9.33 \pm 1.24 \mathrm{~mm} \\
\Phi=10 \pm 0 \mathrm{~mm} \\
\Phi=9.5 \pm 0.4 \mathrm{~mm}\end{array}$ & [1] \\
\hline Aerial parts & Essential oil & $\begin{array}{l}\text { Agar diffusion methods, broth } \\
\text { microdilution assay }\end{array}$ & $\begin{array}{c}\text { Staphylococcus aureus } \\
\text { Escherichia coli } \\
\text { Pseudomonas aeruginosa }\end{array}$ & $\begin{array}{c}\Phi=35.67 \pm 0.66 \mathrm{~mm} \\
M I C=0.03 \% \\
M M C=0.13 \% \\
\Phi=26.33 \pm 1.66 \mathrm{~mm} \\
M I C=0.03 \% \\
M M C=0.03 \% \\
\Phi=9.33 \pm 0.66 \mathrm{~mm} \\
M I C=0.5 \% \\
M M C=0.5 \%\end{array}$ & {$[3]$} \\
\hline Leaves & Ethanolic extract & Agar-well diffusion assay & Escherichia coli & $\Phi=30.33 \pm 2.51 \mathrm{~mm}$ & {$[11]$} \\
\hline Leaves & Essential oil & $\begin{array}{l}\text { Disk-diffusion assay } \\
\text { Microtitration method }\end{array}$ & $\begin{array}{c}\text { Salmonella CECT } 915 \\
\text { Salmonella S64 } \\
\text { E. coli O157: H7 CECT4267 } \\
\text { L. monocytogenes } \\
\text { CECT4031 } \\
\\
\text { L. monocytogenes L23 }\end{array}$ & $\begin{array}{c}\Phi=34.33 \pm 4.04 \mathrm{~mm} \\
M I C=0.0625 \% \\
M B C=0.0625 \% \\
\Phi=28.17 \pm 1.61 \mathrm{~mm} \\
M I C=0.0625 \% \\
M B C=0.125 \% \\
\Phi=19.67 \pm 1.15 \mathrm{~mm} \\
M I C=0.25 \% \\
M B C=0.5 \% \\
\Phi=34.00 \pm 0.00 \mathrm{~mm} \\
M I C=0.125 \% \\
M B C=0.0625 \% \\
\Phi=31.00 \pm 3.46 \mathrm{~mm} \\
M I C=0.5 \% \\
M B C=0.5 \%\end{array}$ & {$[6]$} \\
\hline
\end{tabular}


TABle 2: Continued.

\begin{tabular}{|c|c|c|c|c|c|}
\hline Use part & Extracts & Used method & Tested strains & Key results & References \\
\hline $\begin{array}{l}\text { Flowering } \\
\text { tops }\end{array}$ & Essential oil & $\begin{array}{l}\text { Disk-diffusion assay } \\
\text { Microtitration method }\end{array}$ & $\begin{array}{c}\text { Salmonella CECT } 915 \\
\text { Salmonella S64 } \\
\text { E. coli O157: H7 CECT4267 } \\
\text { L. monocytogenes } \\
\text { CECT4031 } \\
\text { L. monocytogenes L23 }\end{array}$ & $\begin{array}{c}\Phi=31.50 \pm 2.78 \mathrm{~mm} \\
M I C=0.0625 \\
M B C=0.125 \% \\
\Phi=28.17 \pm 1.61 \mathrm{~mm} \\
M I C=0.0625 \% \\
M B C=0.125 \% \\
\Phi=18.00 \pm 0.00 \mathrm{~mm} \\
M I C=0.25 \% \\
M B C=0.5 \% \\
\Phi=29.00 \pm 1.73 \mathrm{~mm} \\
M I C=0.125 \% \\
M B C=0.25 \% \\
\Phi=31.00 \pm 3.46 \mathrm{~mm} \\
M I C=0.5 \% \\
M B C=0.5 \%\end{array}$ & [6] \\
\hline $\begin{array}{l}\text { Flowering } \\
\text { tops }\end{array}$ & Essential oil & & $\begin{array}{l}\text { Total mesophilic aerobic } \\
\text { flora (FMAT) }\end{array}$ & $\begin{array}{l}\text { Significant effect on } \\
\text { microbial growth }\end{array}$ & [10] \\
\hline Aerial parts & Essential oil & $\begin{array}{l}\text { Agar-well diffusion method } \\
\text { Microdilution assay }\end{array}$ & $\begin{array}{l}\text { E. coli ATCC } 25922 \\
\text { E. coli K12 } \\
\text { B. subtilis DCM } 6633 \\
\text { S. aureus ATCC } 25923\end{array}$ & $\begin{array}{c}\Phi=21.33 \pm 0.57 \mathrm{~mm} \\
M I C=0.5 \% \\
M B C<1 \% \\
\Phi=16.00 \pm 1.00 \mathrm{~mm} \\
M I C=0.25 \% \\
M B C=0.5 \% \\
\Phi=24.66 \pm 1.52 \mathrm{~mm} \\
M I C=0.5 \% \\
M B C=0.5 \% \\
\Phi=27.00 \pm 1.73 \mathrm{~mm} \\
M I C=0.125 \% \\
M B C=0.125 \%\end{array}$ & [4] \\
\hline Aerial parts & $\begin{array}{l}\text { Dissolved volatile } \\
\text { fraction }\end{array}$ & $\begin{array}{l}\text { Agar-well diffusion method } \\
\text { Microdilution assay }\end{array}$ & $\begin{array}{l}\text { E. coli ATCC } 25922 \\
\text { E. coli K12 } \\
\text { B. subtilis DCM } 6633 \\
\text { S. aureus ATCC } 25923\end{array}$ & $\begin{array}{c}\Phi=28.33 \pm 0.57 \mathrm{~mm} \\
M I C=0.125 \% \\
M B C=0.25 \% \\
\Phi=17.00 \pm 1.73 \mathrm{~mm} \\
M I C=0.125 \% \\
M B C=0.125 \% \\
\Phi=41.00 \pm 2.64 \mathrm{~mm} \\
M I C=0.0625 \% \\
M B C=0.0625 \% \\
\Phi=30.00 \pm 2.00 \mathrm{~mm} \\
M I C=0.0312 \% \\
M B C=0.0312 \%\end{array}$ & [4] \\
\hline
\end{tabular}

TABle 3: Antifungal activity of O. elongatum.

\begin{tabular}{lcccc}
\hline Use part & Extracts & Used method & Tested strains & Key results \\
\hline Aerial parts & Essential oil & Agar plug diffusion method & $\begin{array}{c}\text { Candida albicans } \\
\text { Aspergillus brasiliensis }\end{array}$ & $\begin{array}{c}\Phi=33.67 \pm 0.33 \mathrm{~mm} \\
\text { No measurable zone of inhibition }\end{array}$ \\
\hline \multirow{2}{*}{ Aerial parts } & Essential oil & Microdilution method & $\begin{array}{c}\text { Candida } \\
\text { Aspergillus } \\
\text { Rhizopus }\end{array}$ & $\begin{array}{c}\text { Sensitive to the essential oil } \\
\text { Susceptible to the oil } \\
\text { Moderately susceptible to the oil }\end{array}$ \\
\hline
\end{tabular}

reported by several investigators (Table 4) [5, 16]. In 2017, Ramzi and collaborators [5] tested the acaricidal activity of the EOs of O. elongatum leaves on the Varroa mite. Therefore, these plant-derived EOs showed certain effectiveness against Varroa. Besides, the antiparasitic effect of $O$. elongatum EO was evaluated in experimental animals (female Wistar rats) infected with 6 Anisakis larvae using the gastric catheter method [16]. This technique was also used to administer O. elongatum (46.9 mg/0.5 mL of olive oil). Consequently, an EO activity against larva L3 of Anisakis pegreffii was observed; moreover, significant alterations of the esophageal region and the cuticle were detected in a large number of recovered larvae. 
TABLe 4: Other activities of O. elongatum.

\begin{tabular}{|c|c|c|c|c|c|}
\hline Activities & Use part & Extracts & Experimental approach & Key results & References \\
\hline \multirow{2}{*}{ Antiviral } & Leaves & Essential oil & $\begin{array}{l}\text { Cytopathogenic murine } \\
\text { norovirus (MNV-1) } \\
\text { RAW } 264.7 \text { cells }\end{array}$ & $0.37 \log _{10} \mathrm{TCID}_{50} / \mathrm{ml}$ reductions & {$[6]$} \\
\hline & $\begin{array}{l}\text { Flowering } \\
\text { tops }\end{array}$ & Essential oil & $\begin{array}{l}\text { Cytopathogenic murine } \\
\text { norovirus (MNV-1) } \\
\text { RAW } 264.7 \text { cells }\end{array}$ & $0.75 \log _{10} \mathrm{TCID}_{50} / \mathrm{ml}$ reductions & {$[6]$} \\
\hline \multirow[b]{2}{*}{ Antiparasitic } & Leaves & Essential oil & $\begin{array}{c}\text { Colonies of Apis mellifera bees } \\
\text { Efficacy against Varroa mite in } \\
\text { beehives }\end{array}$ & $\begin{array}{l}\text { Significant increase in mite drop } \\
\text { All Varroa mites died }\end{array}$ & {$[5]$} \\
\hline & Leaves & Essential oil & $\begin{array}{c}\text { Larva L3 of Anisakis pegreffii } \\
\text { isolated from the host Scomber } \\
\text { japonicas and Trachurus } \\
\text { trachurus } \\
\text { Female Wistar rats infected with } \\
6 \text { Anisakis larvae by gastric } \\
\text { catheter } \\
\text { Administration of } O \text {. elongatum } \\
\text { (46.9 mg/0.5 mL of olive oil) }\end{array}$ & $\begin{array}{l}\text { Significant larvicidal activity } \\
\text { Significant alterations in the } \\
\text { esophageal region and cuticle } \\
\text { detected in a large number of } \\
\text { recovered larvae }\end{array}$ & {$[16]$} \\
\hline Antioxidant & Leaves & Essential oil & $\mathrm{EC}_{50}=1.20 \mathrm{~g}$ of extract $/ \mathrm{g} \mathrm{DPPH}$ & & {$[1]$} \\
\hline Vasodilatory activity & Leaves & $\begin{array}{l}\text { Methanol } \\
\text { extract }\end{array}$ & $\begin{array}{l}\text { Perfusion pressure (PP) of the } \\
\text { mesenteric bed of the rat } \\
\text { Synthesis inhibitor endothelial } \\
\text { vasodilators factors } \\
\text { Vasoconstrictor } \alpha \text {-mimetic: } \\
\text { phenylephrine (PHE) } \\
\text { Difference between the blood } \\
\text { pressure before injection and } \\
\text { blood pressure after injection }\end{array}$ & $\begin{array}{l}\text { Vasodilatory activity } \\
\quad(\mathrm{PP}=50 \mathrm{mmHg})\end{array}$ & {$[17]$} \\
\hline Vasodilatory activity & Leaves & $\begin{array}{l}\text { Ethyl acetate } \\
\text { extract }\end{array}$ & $\begin{array}{l}\text { Perfusion pressure (PP) of the } \\
\text { mesenteric bed of the rat } \\
\text { Synthesis inhibitor endothelial } \\
\text { vasodilators factors } \\
\text { Vasoconstrictor } \alpha \text {-mimetic: } \\
\text { phenylephrine (PHE) } \\
\text { Difference between the blood } \\
\text { pressure before injection and } \\
\text { blood pressure after injection }\end{array}$ & $\begin{array}{l}\text { Vasodilatory activity } \\
\quad(\mathrm{PP}=20 \mathrm{mmHg})\end{array}$ & {$[17]$} \\
\hline $\begin{array}{l}\text { Effect on pomegranate } \\
\text { juice quality }\end{array}$ & $\begin{array}{l}\text { Flowering } \\
\text { tops }\end{array}$ & Essential oil & $\begin{array}{c}\mathrm{pH} \text { variation } \\
\text { Determination of total sugars } \\
\text { Growth of natural flora in the } \\
\text { pomegranate juice }\end{array}$ & $\begin{array}{c}\text { Improved the juice conservation } \\
\text { process while preserving the } \\
\text { nutritional and organoleptic } \\
\text { qualities }\end{array}$ & {$[10]$} \\
\hline Corrosion inhibition & $\begin{array}{l}\text { Leaves and } \\
\text { flowers }\end{array}$ & $\begin{array}{l}\text { Methanol/ } \\
\text { chloroform } \\
\text { extract }\end{array}$ & $\begin{array}{l}\text { Corrosion current density } \\
\text { (jcorr) } \\
\text { Electrochemical measurements } \\
\text { Electrochemical impedance } \\
\text { spectroscopy (EIS) } \\
\text { Mass loss method } \\
\text { Adsorption isotherms }\end{array}$ & $\begin{array}{c}\text { Corrosion potential (ecorr) } \\
\text { decreased from }-399.446 \mathrm{mV} / \\
\text { ESC to }-365.607 \mathrm{mV} / \mathrm{ESC} \\
\text { Significant decrease in corrosion } \\
\text { current (jcorr) } \\
\text { Increased the charge transfer } \\
\text { resistance (Rct) with increased } \\
\text { OEE concentration } \\
\text { Ability of OEE to act as a } \\
\text { protective layer against corrosion } \\
\text { on mild steel }\end{array}$ & {$[18]$} \\
\hline $\begin{array}{l}\text { Hepatoprotective effect } \\
\text { against carbon } \\
\text { tetrachloride }(\mathrm{CCl} 4)\end{array}$ & Leaves & $\begin{array}{l}\text { Methanol } \\
\text { extract }\end{array}$ & $\begin{array}{c}\text { Single-dose intraperitoneal } \\
\text { injection of carbon tetrachloride } \\
(\mathrm{CCl} 4)(0.6 \mathrm{ml} / \mathrm{kg}) \text { induced } \\
\text { hepatotoxicity in rats } \\
\text { Rats treated orally by gavage } \\
\text { four doses of } 250,500,1000 \text {, and } \\
2000 \mathrm{mg} / \mathrm{kg} \text { body weight }\end{array}$ & $\begin{array}{c}\text { Significant }(P<0.0001) \text { decrease } \\
\text { in serum aminotransferase levels } \\
\text { and canalicular enzyme } \\
\text { ALP reduced the architectural } \\
\text { destruction cells }\end{array}$ & {$[19]$} \\
\hline
\end{tabular}


3.9. Antiviral Activity. The O. elongatum EOs, cultivated in northern Morocco, were studied for the inactivation of Murine norovirus (MNV-1) (Table 4), which is a human norovirus surrogate. Interestingly, the EOs from leaves and flowering tops showed antiviral activities of $0.87-0.50$ $\log _{10} \mathrm{TCID}_{50} / \mathrm{mL}$ reduction and $0.75 \log _{10} \mathrm{TCID}_{50} / \mathrm{mL}$ reduction, respectively [6].

3.10. Other Biological Activities. Besides the antiparasitic and antiviral activities, the antioxidant effect of endemic O. elongatum was examined in the Rif in northern of Morocco (Table 4) [1, 17]. The authors evaluated the vasodilator activity of $O$. elongatum leaves, extracted by methanol and ethyl acetate on a Wistar rat mesenteric vascular bed precontracted with norepinephrine. Measurement of the perfusion pressure of the rat mesenteric bed revealed that the methanolic extracts $(\mathrm{PP}=50 \mathrm{mmHg})$ gave more active substances than the leaves of $O$. elongatum extracted in ethyl acetate $(\mathrm{PP}=20 \mathrm{mmHg})$ [17]. Furthermore, the food conservation aspect of flowering tops of the studied plant on fresh pomegranate juice was demonstrated [10]. The findings demonstrated that the EOs combined with heat reduce the growth of natural flora presented in the pomegranate juice and thus improve the juice conservation process, while nutritional and organoleptic qualities were also preserved. Moreover, the impact against corrosion inhibition of $O$. elongatum leaves and flowers, using a mixture of methanol/chloroform, was deeply elaborated by applying the electrochemical impedance spectroscopy (EIS), mass loss method, and adsorption isotherms method [18]. According to their electrochemical parameters measurement, it was shown that the use of extracts decreased the corrosion potential (Ecorr), which ranged from $-399.446 \mathrm{mV} / \mathrm{ESC}$ to $-365.607 \mathrm{mV} / \mathrm{ESC}$. A similar decrease was observed with corrosion current (jcorr). Nonetheless, a significant increase of charge transfer resistance (Rct) was noted, with the increase of the OEE concentration (the ability of layer protection from corrosion on the mild steel) [18]. Another important impact of O. elongatum was surveyed by Douhri et al. [19].

Scientists have shown the hepatoprotective effect of methanolic leaf extracts of this species at different doses against the toxicity induced by carbon tetrachloride $\left(\mathrm{CCl}_{4}\right)$ in rats. The biochemical examination of serum hepatic biomarkers showed a significant decrease in serum aminotransferase levels, the canalicular enzyme, and alkaline phosphatase and reduction in the destruction of hepatic cell architecture at the dose of $2000 \mathrm{mg} / \mathrm{kg} / \mathrm{d}$ [19].

3.11. Toxicology. O. elongatum is an aromatic plant well known for its flavor and widely consumed in Morocco as a condiment and food preservative [24]. From a toxicological point of view, only one study investigated the toxicological properties of $O$. elongatum extract by evaluating acute oral toxicity [19]. The results showed a slight change in behavior with loss of appetite and temporary sedation without any change in pathophysiological and neurological activity with an $\mathrm{LD}_{50}$ greater than $3000 \mathrm{mg} / \mathrm{kg}$. Jenner et al. [27] also reported the same signs in rats when testing carvacrol, the major component of $O$. elongatum, in an acute oral toxicity test. The $\mathrm{LD}_{50}$ in this test was $810 \mathrm{mg} / \mathrm{kg}$, suggesting that carvacrol is the active ingredient responsible for the behavioral change caused by this plant [27].

\section{Biological Mechanism Insights into O. elongatum Main Compounds}

The potent anti-inflammatory activity of the extracts encouraged the authors to isolate the main compounds (thymol, carvacrol, limonene, $\alpha$-pinene, and linalool), which might be responsible for the anti-inflammatory effect. The reported studies showed that thymol inhibits inducible lymphocyte proliferation [28], reduces edema and leukocyte influx to injured areas [29], and induces membrane stabilization (84.11\%) in human red blood cell membrane stabilization assay [30]. Moreover, this molecule had inhibitory effects on various inflammatory mediators such as IL- $1 \beta$, IL- 6 , TNF- $\alpha$, and TNF- $\beta$ [31]. It also decreased c-Fos, NFAT-1, and NFAT-2 expression, with inhibition of inducible phospho-SAPK/JNK and phosphoSTAT3 levels [32]. Molecular investigations showed that thymol also inhibits TLR4 upregulation and suppresses IKK, $\mathrm{i} \kappa \mathrm{b} \alpha$, and p65 phosphorylation [33]. Additionally, carvacrol has also shown an anti-inflammatory effect [34]. This compound was reported to be able to inhibit the production of PGE2 (inflammatory mediator catalyzed by COX1) and suppress COX-2 promoter activity by activating PPAR $\alpha$ and PPAR $\gamma$. It also reduced the expression of LPS-induced COX2 mRNA and protein, suggesting that the action of carvacrol on COX-2 is mediated through its agonistic effect on PPAR $\gamma$ [35]. Moreover, Yoon et al. [36] showed that limonene decreased the production of proinflammatory cytokines and inflammatory mediators in macrophages by the inhibition of LPS-induced NO and PGE2, which decreased iNOS and COX-2 expression [36]. This monoterpene also exhibited an anti-inflammatory effect via the inhibition of some signaling pathways leading to the inflammatory process in leukemia (HL-60) cell lines, such as ROS, monocyte chemoattractant protein-1 (MCP-1), NF- $\kappa$ B, and p38 mitogen-activated protein kinase (MAPK) [37]. On the other hand, $\alpha$-pinene exhibited potent activity in the inflammatory process and neuropathic pain [38]. It inhibited ear edema at $0.15 \mathrm{~h}$ (120-135\% vs. $175 \%)$, paw edema at $12 \mathrm{~h}(146 \pm 6 \%)$, and a decrease in COX-2 (115 $\pm 74 \%$ vs. $202 \pm 20 \%)$ [39] and reduced the level of IL- 6 in the hippocampus, cortex, and striatum [40]. In addition, linalool is another monoterpene, which also exhibited an anti-inflammatory effect. This compound significantly reduced hypersensitivity and paw edema at doses of 50 and $200 \mathrm{mg} / \mathrm{kg}$ of carrageenan-induced edema model in rats [41, 42].

Several studies showed the antidiabetic effect of different compounds identified in O. elongatum. The authors showed that carvacrol exhibits antidiabetic effects via several mechanisms such as reduction in blood glucose and insulin levels, decrease in (HOMA-IR) index, and decrease in the expressions of the mRNA of gluconeogenic genes, PEPCK, and G6Pase [43]. Additionally, carvacrol may also decrease 


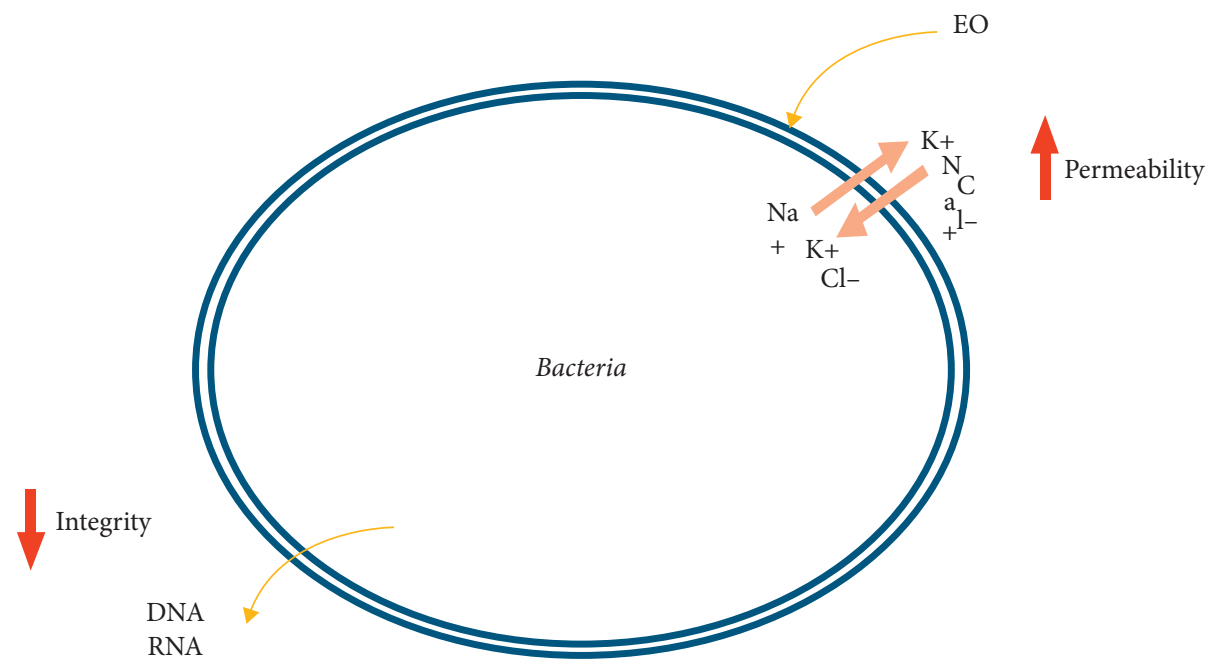

FIgUre 3: Antibacterial action of O. elongatum bioactive compounds.

glucose levels by lowering $\mathrm{HbA}_{1 \mathrm{c}}$, G6Pase, and FBPase activities. It also promoted the activities of glucokinase and glucose-6-phosphate dehydrogenase in the liver and protected pancreatic islets [44]. This monoterpene inhibited the activity of $\alpha$-amylase $\left(\mathrm{IC}_{50}=152.3 \pm 1.21 \mu \mathrm{g} \mathrm{mL}^{-1}\right.$ ), $\alpha$-glucosidase $\left(\mathrm{IC}_{50}=94.02 \pm 0.78 \mu \mathrm{g} \mathrm{mL}^{-1}\right)$ [45], and $\beta$-galactosidase [46]. On the other hand, limonene ameliorates glucose homeostasis by increasing hepatic glycogen with a decrease in plasma glucose and $\mathrm{HbA}_{1 \mathrm{c}}$ levels and suppresses the activities of gluconeogenic enzymes (G6Pase and FBPase) [47]. Moreover, it ameliorates the reduction of FBG level and glucose tolerance along with the activation of PPAR $\alpha$ signaling [48]. In addition, using two different cell lines, C2C12 skeletal muscle cells [49] and 3T3-L1 preadipocytes [50], limonene has been shown to improve glucose absorption by increasing phosphorylation of activated protein kinase $\mathrm{B}$ (Akt) and promoting p38 mitogenactivated protein kinase (p38MAPK) [50].

Furthermore, linalool also showed an antidiabetic effect $[51,52]$. This compound was reported to be able to decrease blood glucose, $\mathrm{HbA}_{1 \mathrm{c}}$, fructosamine, IL-6 and TNF- $\alpha$, and area under the curve of $\left(\mathrm{AUC}_{\mathrm{glucose}}\right)$ glucose value and increase insulin level [52]. On the other hand, thymol was also able to treat hyperglycemia by normalizing blood sugar, plasma insulin, $\mathrm{HbA}_{1 \mathrm{c}}$, and insulin resistance index [53]. Rhayour et al. [54] investigated the expression levels of genes involved in insulin transcription in STZ-induced diabetic rats and reported an increase in expression of the Mafa and $P d x 1$ genes.

The major compounds of this plant are limonene, linalool, carvacrol, and thymol. In fact, these molecules have shown in some studies a significant antibacterial power [55-57]. Rhayour et al. [58] examined the mechanism of action of thymol on bacteria E. coli and Bacillus subtilis as the model of Gram-positive and Gram-negative bacteria. This action was demonstrated by the release of absorbent substances at $260 \mathrm{~nm}$. This release of substances associated with rapid bacterial mortality could be the consequence of lesions on the envelopes induced by antibacterial agents (Figure 3).
Another study [59] showed that carvacrol affects cell membranes of bacteria by changing the composition of fatty acids, which subsequently affects the fluidity and permeability of the membrane. On the other hand, several studies have indicated that linalool alters normal cell morphology, destroys the cell wall and cell membrane, inhibits the growth of $P$. aeruginosa, and even leads to its death [60]. However, the mechanism of action of limonene against cytoplasmic membranes of microorganisms results in a loss of membrane integrity (Figure 3), inhibition of respiratory enzymes, and dissipation of the proton motive force [61].

Numerous studies have been published on the anticancer activity of the main compounds of oregano EOs such as limonene, carvacrol, and thymol [62-64]. Islam et al. [63] determined that the mechanism of action of thymol in a cancer cell caused severe DNA damage through several mechanisms (e.g., ROS induction and subsequent increase in oxidative stress and/or mitochondrial dysfunction or nuclear factor of activated T-cells (NFAT-2) pathway), which eventually upregulates $\mathrm{Bax} / \mathrm{Bcl}-2$ protein expression and results in the cytochrome- (cyto-) c release from the mitochondria (intrinsic pathway). In another work, carvacrol treatment induced cell apoptosis, possibly through the activation of the mitochondrial apoptotic, MAPK, and PI3K/ Akt signaling pathways. Taken together, our results indicate that carvacrol might be a promising natural product in the management of colon cancer [65].

\section{Conclusion}

Morocco is a country rich in plant resources with a specific diversity of medicinal plants used in the treatment and prevention of several illnesses. This study provides evidence that the Moroccan O. elongatum L. species possesses active principles that exhibit marked therapeutic effects confirming and justifying the popular uses of these plants to treat certain diseases as antibacterial, antifungal, antiviral, antioxidant, vasodilator, corrosion inhibitor, and hepatoprotective agents. The current study represents useful documentation 
that can provide sufficient support for clinical trials of O. elongatum L. Although preliminary studies have confirmed their therapeutic effect, further investigations should be carried out, in particular, to ensure the safety of the treatment.

\section{Data Availability}

The data used to support the findings of this study are included within the article.

\section{Conflicts of Interest}

The authors declare that they have no conflicts of interest.

\section{Authors' Contributions}

Abdelaali Balahbib and Abdelhakim Bouyahya contributed equally to this work.

\section{References}

[1] H. Oualili, R. Nmila, and F. Chibi, "Chemical composition and antioxidant activity of Origanum elongatum essential oil,” Pharmacognosy Research, vol. 11, no. 3, p. 283, 2019.

[2] M. Bakha, C. Al Faiz, M. Daoud et al., "Genome size and chromosome number for six taxa of Origanum genus from Morocco," Botany Letters, vol. 164, no. 4, pp. 361-370, 2017.

[3] S. Boukhira, F. Bousta, S. Moularat, A. Abdellaoui, Z. B. Ouaritini, and D. Bousta, "Evaluation of the preservative properties of origanum elongatum essential oil in a topically applied formulation under a challenge test," Phytothérapie, vol. 18, no. 2, pp. 92-98, 2018.

[4] A. El Harsal, A. Ibn Mansour, N. Skali Senhaji et al., "Influence of extraction time on the yield, chemical composition, and antibacterial activity of the essential oil from origanum elongatum (E. \& M.) harvested at Northern Morocco," Journal of Essential Oil Bearing Plants, vol. 21, no. 6, pp. 1460-1474, 2018.

[5] H. Ramzi, M. R. Ismaili, M. Aberchane, and S. Zaanoun, "Chemical characterization and acaricidal activity of Thymus satureioides C. \& B. and Origanum elongatum E. \& M. (Lamiaceae) essential oils against Varroa destructor Anderson \& Trueman (Acari: Varroidae)," Industrial Crops and Products, vol. 108, pp. 201-207, 2017.

[6] N. Moussaoui, G. Sanchez, and E. O. Khay, "Antibacterial and antiviral activities of essential oils of northern moroccan plants," British Biotechnology Journal, vol. 3, no. 3, pp. 318-331, 2013.

[7] G. Figuérédo, P. Cabassu, J.-C. Chalchat, and B. Pasquier, "Studies of mediterranean oregano populations. vi: chemical composition of essential oils of origanum elongatumemberger et maire from Morocco," Journal of Essential Oil Research, vol. 18 , no. 3, pp. 278-280, 2006.

[8] G. Figueredo, "Etude chimique et statistique de la composition d'huiles essentielles d'origans (Lamiaceae) cultivés issus de graines d'origine méditerranéenne," Université Blaise Pascal-Clermont-Ferrand II, Clermont-Ferrand, France, Theses, 2007.

[9] H. Bouharb, K. El Badaoui, T. Zair, J. El amri, S. Chakir, and T. Alaoui, "Sélection de quelques plantes médicinales du Zerhoun (Maroc centrale) pour l'activité antibactérienne contre Pseudomonas aeruginosa," Journal of Applied Biosciences, vol. 78, pp. 6685-6693, 2014.

[10] N. E. Moussaoui, E. O. Khay, N. Amajoud et al., "Effect of Origanum elongatum essential oil and heating on pomegranate juice quality," International Journal of Current Microbiology and Applied Sciences, vol. 5, no. 4, pp. 1-8, 2016.

[11] H. Douhri, I. Raissouni, N. Amajoud et al., "Antibacterial effect of ethanolic extracts of Moroccan plant against Escherichia coli," Journal of Materials and Environmental Sciences, vol. 8, no. 12, pp. 4408-4414, 2017.

[12] A. Amakran, M. Hamoudane, and B. Ramdan, "Antifungal activity of the essential oil of origanum elongatum on candida, aspergillus and rhizopus species," Journal de Mycologie Medicale, vol. 2, no. 24, p. e78, 2014.

[13] S. Coskun, O. Girisgin, M. Kürkcüoglu et al., “Acaricidal efficacy of Origanum onites L. essential oil against Rhipicephalus turanicus (Ixodidae)," Parasitology Research, vol. 103, no. 2, pp. 259-261, 2008.

[14] S. Degerli, B. Tepe, A. Celiksoz, S. Berk, and E. Malatyali, "In vitro amoebicidal activity of Origanum syriacum and Origanum laevigatum on Acanthamoeba castellanii cysts and trophozoites," Experimental Parasitology, vol. 131, no. 1, pp. 20-24, 2012.

[15] M. Machado, A. M. Dinis, L. Salgueiro, C. Cavaleiro, J. B. A. Custódio, and M. d. C. Sousa, "Anti-Giardia activity of phenolic-rich essential oils: effects of Thymbra capitata, Origanum virens, Thymus zygis subsp. sylvestris, and Lippia graveolens on trophozoites growth, viability, adherence, and ultrastructure," Parasitology Research, vol. 106, no. 5, pp. 1205-1215, 2010.

[16] N. Abattouy, A. Valero López, and M. C. Romero, “Actividad IN VIVO del aceite esencial de Origanum elongatum frente a larvas L3 de Anisakis pegreffii," IN VIVO Activity of Essential Oil Origanum Elongatum Against Larva L3 of Anisakis Pegreffi, vol. 51, pp. 107-111, 2010.

[17] K. E. Yousfi, H. Greche, H. Misbahi, and R. B. Cheikh, "Phytochemical evaluation and vasodilatory activity of O. elongatum, C. salviifolius and C. laurifolius," Moroccan Journal of Chemistry, vol. 8, no. 1, pp. 226-234, 2020.

[18] H. El Attari, K. Chefira, and H. Rchid, "Corrosion inhibition study of the origanum elongatum extract: electrochemical, gravimetric and adsorption isotherms studies in $0.5 \mathrm{~m}$ sulfuric acid," Research Journal of Pharmaceutical, Biological and Chemical Sciences, vol. 10, no. 1, 2019.

[19] B. Douhri, M. Idaomar, N. S. Senhaji, A. Ennabili, and J. Abrini, "Hepatoprotective effect of origanum elongatum against Carbon Tetrachloride (CCl4) induced toxicity in rats," European Journal of Medicinal Plants, vol. 4, no. 1, pp. 14-28, 2014.

[20] J. H. Ietswaart and V. Else, A Taxonomic Revision of the Genus Origanum (Labiatae), The Hague: Leiden University Press, The Hague, The Netherlands, 1980.

[21] S. E. Kintzios, "Oregano," in In Handbook of Herbs and Spices, K. V. Peter, Ed., pp. 417-436, Woodhead Publishing, Sawston, UK, 2nd edition, 2012.

[22] X. Simonnet, M. Quennoz, D. Bellenot, and B. Pasquier, "Evaluation agronomique et chimique de différentes espèces d'origan," Revue suisse de viticulture, arboriculture et horticulture, vol. 43, no. 6, pp. 344-349, 2011.

[23] Euro+MedPlantBase, "The information resource for euromediterranean plant diversity," 2006, http://ww2.bgbm.org/ EuroPlusMed/query.asp.

[24] E. Christaki, E. Bonos, I. Giannenas, and P. Florou-Paneri, "Aromatic plants as a source of bioactive compounds," $\mathrm{Ag}$ riculture, vol. 2, no. 3, pp. 228-243, 2012. 
[25] A. Benabid, "Flore et ecosystemes du Maroc: evaluation et preservation de la biodiversite," 2000.

[26] O. Belmehdi, A. El Harsal, M. Benmoussi, Y. Laghmouchi, N. Skali Senhaji, and J. Abrini, "Effect of light, temperature, salt stress and $\mathrm{pH}$ on seed germination of medicinal plant Origanum elongatum (Bonnet) Emb. \& Maire," Biocatalysis and Agricultural Biotechnology, vol. 16, pp. 126-131, 2018.

[27] P. M. Jenner, E. C. Hagan, J. M. Taylor, E. L. Cook, and O. G. Fitzhugh, "Food flavourings and compounds of related structure I. acute oral toxicity," Food and Cosmetics Toxicology, vol. 2, pp. 327-343, 1964.

[28] Z. Amirghofran, R. Hashemzadeh, K. Javidnia, H. Golmoghaddam, and A. Esmaeilbeig, "In vitroimmunomodulatory effects of extracts from three plants of theLabiataefamily and isolation of the active compound(s)," Journal of Immunotoxicology, vol. 8, no. 4, pp. 265-273, 2011.

[29] M. E. Pascual, K. Slowing, E. Carretero, D. Sánchez Mata, and A. Villar, "Lippia: traditional uses, chemistry and pharmacology: a review," Journal of Ethnopharmacology, vol. 76, no. 3, pp. 201-214, 2001.

[30] J. Sheorain, M. Mehra, R. Thakur, S. Grewal, and S. Kumari, "In vitro anti-inflammatory and antioxidant potential of thymol loaded bipolymeric (tragacanth gum/chitosan) nanocarrier," International Journal of Biological Macromolecules, vol. 125, pp. 1069-1074, 2019.

[31] Y.-M. Yu, T.-Y. Chao, W.-C. Chang, M. J. Chang, and M.-F. Lee, "Thymol reduces oxidative stress, aortic intimal thickening, and inflammation-related gene expression in hyperlipidemic rabbits," Journal of Food and Drug Analysis, vol. 24 , no. 3, pp. 556-563, 2016.

[32] N. Gholijani, M. Gharagozloo, S. Farjadian, and Z. Amirghofran, "Modulatory effects of thymol and carvacrol on inflammatory transcription factors in lipopolysaccharidetreated macrophages," Journal of Immunotoxicology, vol. 13, no. 2, pp. 157-164, 2016.

[33] Q. Wang, F. Cheng, Y. Xu et al., "Thymol alleviates lipopolysaccharide-stimulated inflammatory response via downregulation of RhoA-mediated NF- $\kappa$ B signalling pathway in human peritoneal mesothelial cells," European Journal of Pharmacology, vol. 833, pp. 210-220, 2018.

[34] P. Landa, L. Kokoska, M. Pribylova, T. Vanek, and P. Marsik, "In vitro anti-inflammatory activity of carvacrol: inhibitory effect on COX-2 catalyzed prostaglandin E2 biosynthesisb," Archives of Pharmacal Research, vol. 32, no. 1, pp. 75-78, 2009.

[35] M. Hotta, R. Nakata, M. Katsukawa, K. Hori, S. Takahashi, and H. Inoue, "Carvacrol, a component of thyme oil, activates $\operatorname{PPAR} \alpha$ and $\gamma$ and suppresses COX-2 expression," Journal of Lipid Research, vol. 51, no. 1, pp. 132-139, 2010.

[36] W.-J. Yoon, N. H. Lee, and C.-G. Hyun, "Limonene suppresses lipopolysaccharide-induced production of nitric oxide, prostaglandin E2, and pro-inflammatory cytokines in RAW 264.7 macrophages," Journal of Oleo Science, vol. 59, no. 8, pp. 415-421, 2010.

[37] D. A. Wiseman, S. R. Werner, and P. L. Crowell, "Cell cycle arrest by the isoprenoids perillyl alcohol, geraniol, and farnesol is mediated by $\mathrm{p} 21$ cipland p27kip 1 in human pancreatic adenocarcinoma cells," Journal of Pharmacology and Experimental Therapeutics, vol. 320, no. 3, pp. 1163-1170, 2007.

[38] N. Quintão, G. da Silva, C. Antonialli, L. Rocha, V. Filho, and J. Cicció, "Chemical composition and evaluation of the antihypernociceptive effect of the essential oil extracted from the leaves of ugni myricoideson inflammatory and neuropathic models of pain in mice," Planta Medica, vol. 76, no. 13, pp. 1411-1418, 2010.
[39] X.-J. Li, Y.-J. Yang, Y.-S. Li, W. K. Zhang, and H.-B. Tang, " $\alpha$-Pinene, linalool, and 1-octanol contribute to the topical anti-inflammatory and analgesic activities of frankincense by inhibiting COX-2," Journal of Ethnopharmacology, vol. 179, pp. 22-26, 2016.

[40] M. Khoshnazar, M. R. Bigdeli, S. Parvardeh, and R. Pouriran, "Attenuating effect of $\alpha$-pinene on neurobehavioural deficit, oxidative damage and inflammatory response following focal ischaemic stroke in rat," Journal of Pharmacy and Pharmacology, vol. 71, no. 11, pp. 1725-1733, 2019.

[41] P. A. Batista, M. F. de Paula Werner, E. C. Oliveira et al., "The antinociceptive effect of (-)-linalool in models of chronic inflammatory and neuropathic hypersensitivity in mice," The Journal of Pain, vol. 11, no. 11, pp. 1222-1229, 2010.

[42] A. T. Peana, P. S. D’Aquila, F. Panin, G. Serra, P. Pippia, and M. D. L. Moretti, "Anti-inflammatory activity of linalool and linalyl acetate constituents of essential oils," Phytomedicine, vol. 9, no. 8, pp. 721-726, 2002.

[43] K. G. Woon, J. H. Kyung, K. Do Yeon, and C. S. Hyun, "Beneficial effects of carvacrol on insulin resistance in high fat diet-induced diabetic mice," in Proceedings of the Fall General Meeting and Academic Conference 2013, p. 281, San Francisco, CA, USA, October 2013.

[44] M. Ezhumalai, T. Radhiga, and K. V. Pugalendi, "Antihyperglycemic effect of carvacrol in combination with rosiglitazone in high-fat diet-induced type 2 diabetic C57BL/6J mice," Molecular and Cellular Biochemistry, vol. 385, no. 1-2, pp. 23-31, 2014.

[45] S. Govindaraju and P. I. Arulselvi, "Characterization of coleus aromaticus essential oil and its major constituent carvacrol forin vitroantidiabetic and antiproliferative activities," Journal of Herbs, Spices \& Medicinal Plants, vol. 24, no. 1, pp. 37-51, 2018.

[46] J. Wang, X. Hu, W. Ai et al., "Phytol increases adipocyte number and glucose tolerance through activation of PI3K/Akt signaling pathway in mice fed high-fat and high-fructose diet," Biochemical and Biophysical Research Communications, vol. 489, no. 4, pp. 432-438, 2017.

[47] R. Murali and R. Saravanan, "Antidiabetic effect of d-limonene, a monoterpene in streptozotocin-induced diabetic rats," Biomedicine \& Preventive Nutrition, vol. 2, no. 4, pp. 269-275, 2012.

[48] L. Jing, Y. Zhang, S. Fan et al., "Preventive and ameliorating effects of citrus d-limonene on dyslipidemia and hyperglycemia in mice with high-fat diet-induced obesity," European Journal of Pharmacology, vol. 715, no. 1-3, pp. 46-55, 2013.

[49] I. Soundharrajan, D. H. Kim, S. Srisesharam, P. Kuppusamy, R. Sivanesan, and K. C. Choi, "Limonene promotes osteoblast differentiation and 2-deoxy- $\mathrm{d}$-glucose uptake through p38MAPK and Akt signaling pathways in C2C12 skeletal muscle cells," Phytomedicine, vol. 45, pp. 41-48, 2018.

[50] I. Soundharrajan, D. H. Kim, S. Srisesharam, P. Kuppusamy, and K. C. Choi, "R-limonene enhances differentiation and 2Deoxy-D-Glucose uptake in 3t3-11 preadipocytes by activating the akt signaling pathway," Evidence-Based Complementary and Alternative Medicine, vol. 2018, pp. 1-10, 2018.

[51] T. A. More, B. R. Kulkarni, M. L. Nalawade, and A. U. Arvindekar, "Antidiabetic activity of linalool and limonene in streptozotocin- induced diabetic rat: a combinatorial therapy approach," International Journal of Pharmacy and Pharmaceutical Sciences, vol. 6, no. 8, p. 6, 2014.

[52] B. Deepa and C. V. Anuradha, "Linalool, a plant derived monoterpene alcohol, rescues kidney from diabetes-induced 
nephropathic changes via blood glucose reduction," Diabetologia Croatica, vol. 17, 2011.

[53] S. Saravanan and L. Pari, "Role of thymol on hyperglycemia and hyperlipidemia in high fat diet-induced type 2 diabetic C57BL/6J mice," European Journal of Pharmacology, vol. 761, pp. 279-287, 2015.

[54] A. Saadat Brujeni, "Thymol effect on the expression of Mafa and $\mathrm{Pdx} 1$ genes in streptozotocin-induced diabetic rats," Razi Journal of Medical Sciences, vol. 26, no. 10, 2019.

[55] K. Hạc-Wydro, M. Flasiński, and K. Romańczuk, "Essential oils as food eco-preservatives: model system studies on the effect of temperature on limonene antibacterial activity," Food Chemistry, vol. 235, pp. 127-135, 2017.

[56] W. Churklam, S. Chaturongakul, B. Ngamwongsatit, and R. Aunpad, "The mechanisms of action of carvacrol and its synergism with nisin against Listeria monocytogenes on sliced bologna sausage," Food Control, vol. 108, Article ID 106864, 2020.

[57] A. Duarte, Â. Luís, M. Oleastro, and F. C. Domingues, "Antioxidant properties of coriander essential oil and linalool and their potential to control Campylobacter spp," Food Control, vol. 61, pp. 115-122, 2016.

[58] K. Rhayour, T. Bouchikhi, A. Tantaoui-Elaraki, K. Sendide, and A. Remmal, "The mechanism of bactericidal action of oregano and clove essential oils and of their phenolic major components onescherichia coliand bacillus subtilis," Journal of Essential Oil Research, vol. 15, no. 4, pp. 286-292, 2003.

[59] M. K. Swamy, M. S. Akhtar, and U. R. Sinniah, "Antimicrobial properties of plant essential oils against human pathogens and their mode of action: an updated review," Evidence-based Complementary and Alternative Medicine, vol. 2016, Article ID 3012462, 2016.

[60] X. Liu, J. Cai, H. Chen et al., "Antibacterial activity and mechanism of linalool against Pseudomonas aeruginosa," Microbial Pathogenesis, vol. 141, Article ID 103980, 2020.

[61] Z. Zhang, F. Vriesekoop, Q. Yuan, and H. Liang, "Effects of nisin on the antimicrobial activity of d-limonene and its nanoemulsion," Food Chemistry, vol. 150, pp. 307-312, 2014.

[62] J. Baranauskaite, A. Kubiliene, M. Marksa et al., "The influence of different oregano species on the antioxidant activity determined using HPLC postcolumn DPPH method and anticancer activity of carvacrol and rosmarinic acid," BioMed Research International, vol. 2017, Article ID 1681392, 2017.

[63] M. T. Islam, A. B. R. Khalipha, R. Bagchi et al., "Anticancer activity of thymol: a literature-based review and docking study with Emphasis on its anticancer mechanisms," IUBMB Life, vol. 71, no. 1, pp. 9-19, 2019.

[64] K. C. Sekhar, A. Rajanikanth, M. N. Bobby, and J. R. Kanala, "A review on anticancer potential of natural drugs: hispolon and limonene," International Journal of Current Microbiology and Applied Sciences, vol. 7, no. 11, pp. 3253-3263, 2018.

[65] K. Fan, X. Li, Y. Cao et al., "Carvacrol inhibits proliferation and induces apoptosis in human colon cancer cells," AntiCancer Drugs, vol. 26, no. 8, pp. 813-823, 2015. 